\title{
"Capillary Electrophoresis Monitors Enhancement in Reactive Oxygen Species Subcellular Production upon Treatment with Doxorubicin"
}

\author{
Angela R Eder, Edgar A Arriaga*
}

*Author to whom correspondence should be addressed. Tel.: +1 612624 8024; fax +1

612626 7541. E-mail address: arriaga@chem.umn.edu

By establishing histograms describing the distribution of organelle electrophoretic mobility for organelles containing ROS, DOX, and both ROS and DOX, it is possible to speculate which organelles correspond to these populations. Table S1 details the mean and median electrophoretic mobility of organelles found in each population.

Table S1. Electrophoretic mobilities of organelles containing ROS, DOX and both ROS and DOX. ${ }^{a}$

\begin{tabular}{|l|c|c|c|c|c|c|}
\hline & Untreated & \multicolumn{6}{|c|}{ DOX Treated } \\
\cline { 2 - 7 } & ROS & ROS & DOX & $\begin{array}{c}\text { DOX \& } \\
\text { ROS (A1l) }\end{array}$ & $\begin{array}{c}\text { DOX \& } \\
\text { ROS (non- } \\
\text { linear) }\end{array}$ & $\begin{array}{c}\text { DOX \& } \\
\text { ROS } \\
\text { (linear) }\end{array}$ \\
\hline Mean & $-5.10^{b} \pm$ & $-5.08 \pm$ & $-4.98 \pm$ & $-3.33 \pm$ & $-1.19 \pm$ & $-1.90 \pm$ \\
& -0.02 & -0.02 & -0.01 & -0.02 & -0.11 & -0.03 \\
\hline Median & $-2.23 \pm$ & $-2.25 \pm$ & $-2.31 \pm$ & $-1.01 \pm$ & $-0.93 \pm$ & $-1.93 \pm$ \\
& -0.10 & -0.06 & -0.07 & -0.02 & -0.03 & -0.02 \\
\hline
\end{tabular}

${ }^{a}$ Electrophoretic mobility $\left(10^{-4}\right)\left(\mathrm{cm}^{2} \mathrm{~V}^{-1} \mathrm{~s}^{-1}\right)$.

${ }^{b}$ Data represent the mean and standard deviation of triplicate analyses. 
Supporting Information

Eder, Arriaga
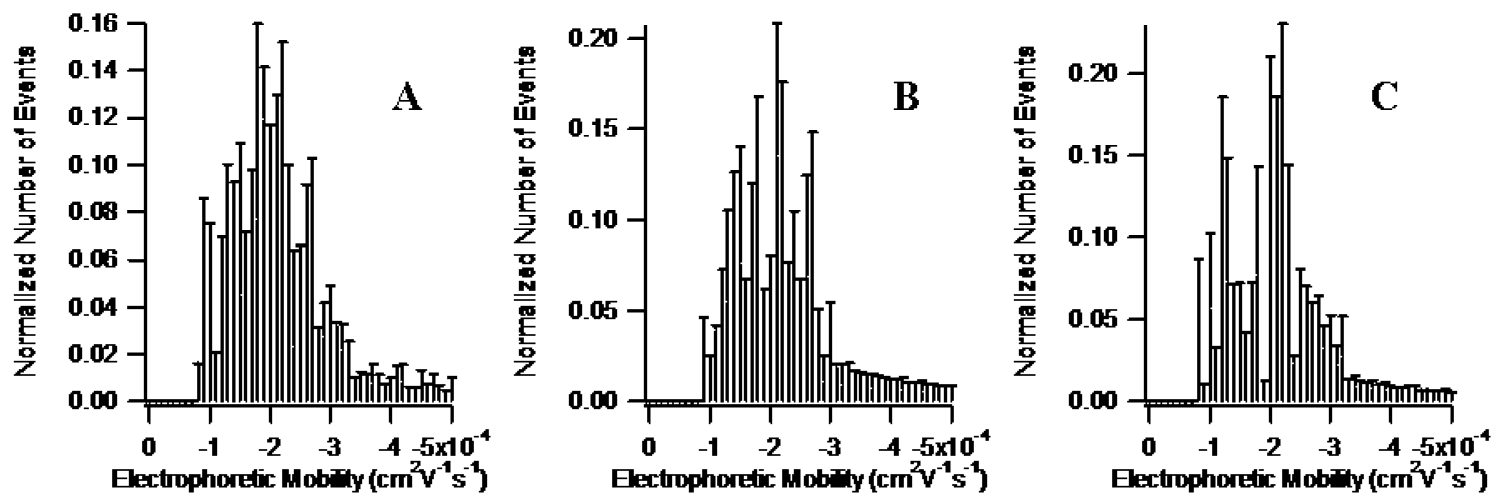

Figure S1. Electrophoretic mobility distributions of individually electrophoresed organelles. (A) Organelles containing DCF from untreated cells (B) organelles containing DCF from DOX-treated cells (C) organelles containing DOX from DOX treated cells.

The organelles in Figure S1 that contain ROS (Figure S1 (A) and (B)) show similar electrophoretic profiles, regardless of their treatment status. However, organelles containing DOX (Figure S1 (C)) have a distinct pattern of electrophoretic mobility when compared to the distributions obtained for organelles containing ROS.

Organelles containing both DOX and ROS are only a subset of those organelles that contain DOX (c.f. Figure S1 (C)). These results imply that there are at least two types of organelles containing DOX: in one of these types, DOX induces ROS production; the other type accumulates DOX but does not induce ROS production. These organelles are likely mitochondria and acidic organelles, respectively. 
Supporting Information

Eder, Arriaga

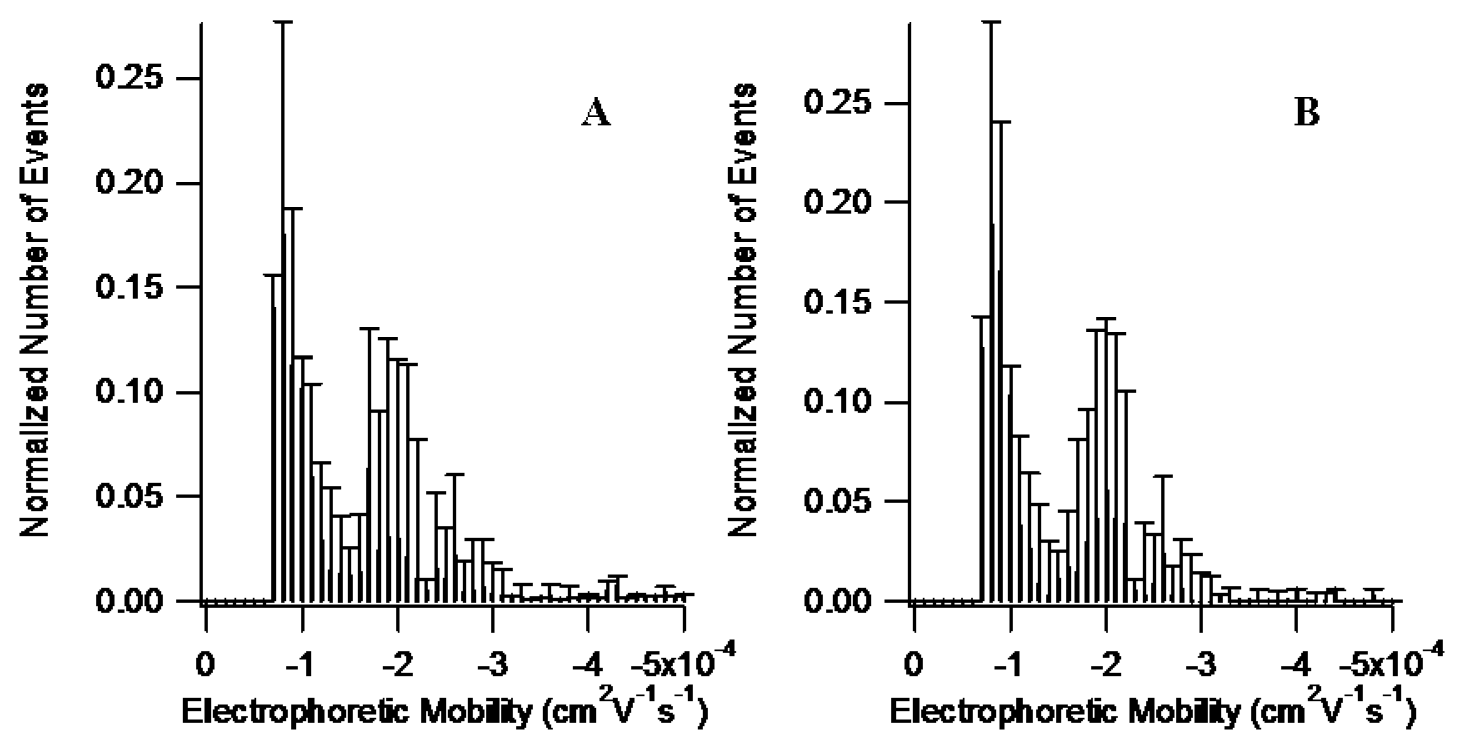

Figure S2. Electrophoretic mobility distribution for all organelles containing DOX. (A) Organelles containing only DOX (B) Organelles containing both DOX and ROS.

Organelles containing both DOX and ROS are only a subgroup of those organelles containing DOX. These results imply that there are at least two types of organelles containing DOX: in one of these types, DOX induces ROS production (Figure S2 (A)); the other type does not have ROS production associated with DOX accumulation (Figure S2 (B)). The bimodal distribution indicates that two types of organelles are present in both distributions. These organelles are likely mitochondria and acidic organelles. 
Supporting Information

Eder, Arriaga

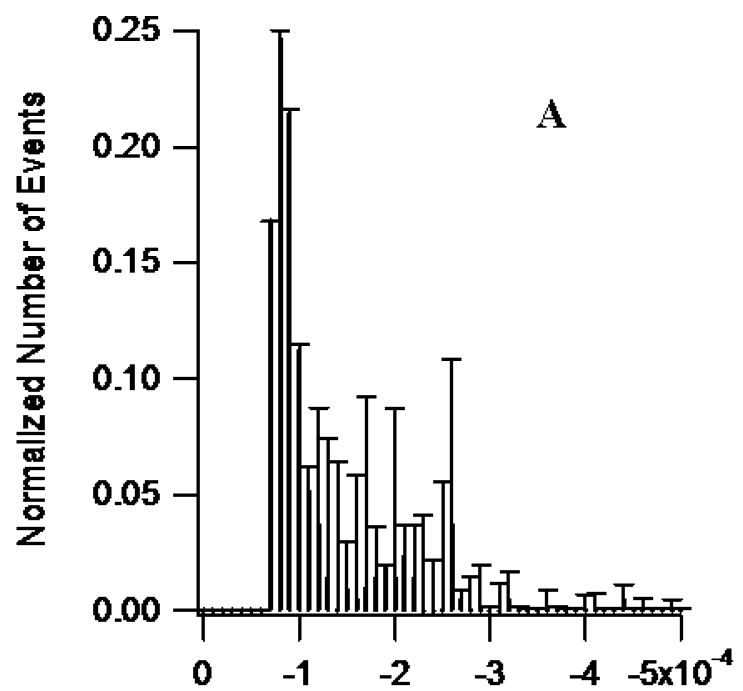

Electrophoretic Mobitiy $\left(\mathrm{cm}^{2} \mathrm{v}^{-1} \mathrm{~s}^{-1}\right)$

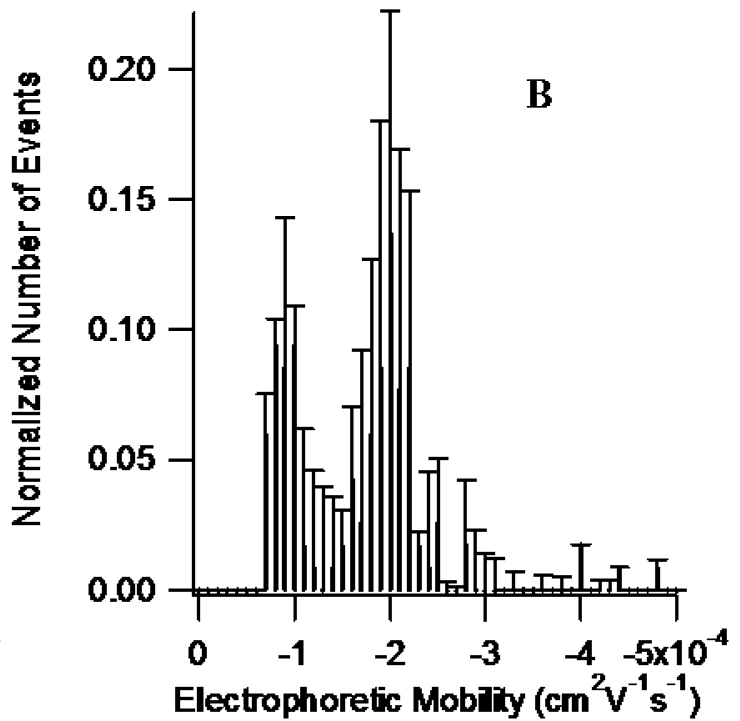

Figure S3. Electrophoretic mobility distribution for organelles containing both DOX and ROS. (A) Organelles containing both DOX and ROS that do not show a linear response between DOX accumulation and ROS production (c.f. Figure 10 (A)). (B) Organelles containing both DOX and ROS demonstrating a direct relationship between DOX accumulation and ROS production (c.f. Figure $10(\mathrm{C})$ ).

Two sub-populations of organelles containing both DOX and ROS were found; one that exhibited no clear relationship between DOX uptake and ROS production (c.f. Figure $10(\mathrm{~B}))$ and another that showed a direct relationship between DOX uptake and ROS production (c.f. Figure $10(\mathrm{C})$ ). To examine further whether these relationships were the result of different organelle types, the electrophoretic mobility distributions for each sub-population were compared (Figure S3). Organelles in which a direct relationship between DOX uptake and ROS production existed had a median electrophoretic mobility of $-1.93 \pm-0.89+10^{-4} \mathrm{~cm}^{2} \mathrm{~V}^{-1} \mathrm{~s}^{-1}$ (Figure S3 (B)), whereas those demonstrating no such relationship had a median electrophoretic mobility of $-0.93 \pm-$ $0.73-10^{-4} \mathrm{~cm}^{2} \mathrm{~V}^{-1} \mathrm{~s}^{-1}$ (Figure S3 (A)). This seems to indicate that DOX-induced ROS production is localized in the mitochondria, as the electrophoretic mobility is similar to 
Supporting Information

Eder, Arriaga

that reported previously. ${ }^{1}$ The other organelle population, which did not exhibit DOX-

induced ROS production, is likely either acidic organelles or peroxisomes.

\section{References:}

1. Fuller, K.; Duffy, C.; Arriaga, A. Electrophoresis, 2002, 23, 1571-1576. 